\title{
Análisis del crecimiento urbano y su relación con el incremento de temperaturas en la ciudad de Mérida utilizando imágenes satelitales
}

\author{
Saul Navarro-Tec ${ }^{1}$, Mauricio Gabriel Orozco-del-Castillo ${ }^{1}$, \\ Juan Carlos Valdiviezo-Navarro ${ }^{2}$, Daniel Rolando Ordaz-Bencomo ${ }^{1}$, \\ Mario Renan Moreno-Sabido ${ }^{1}$, Carlos Bermejo-Sabbagh ${ }^{1}$ \\ ${ }^{1}$ Instituto Tecnológico de Mérida, \\ Departamento de Sistemas y Computación, Yucatán, \\ México \\ ${ }^{2}$ Centro de Investigación en Ciencias de Información Geoespacial, Unidad Mérida, Yucatán, \\ México \\ \{saulnavarrotec, daniel.ordaz9323, xacdc12\}@gmail.com, \\ mauricio.orozco@itmerida.edu.mx, \\ jvaldiviezo@centrogeo.edu.mx, cbermejo00@hotmail.com
}

\begin{abstract}
Resumen. En este artículo se realiza un análisis de la correlación entre el crecimiento de la mancha urbana y el cambio de temperaturas de la ciudad de Mérida, Yucatán, México, mediante la implementación de técnicas de inteligencia artificial enfocadas a la segmentación de imágenes. Partiendo de una secuencia multitemporal de imágenes satelitales registradas por Landsat en formato RGB ocupando un rango de los años 2001 al 2016, se realiza la segmentación de la mancha urbana utilizando una técnica de inteligencia artificial, particularmente optimización por enjambre de partículas, una implementación de inteligencia de enjambres. La segmentación de los datos nos permite estimar el historial de crecimiento del área de suelo construido en la ciudad. Posteriormente los datos históricos de temperaturas registradas en ese mismo periodo son analizados con el método de descomposición modal empírica. El análisis preliminar de la correlación positiva entre los datos de área construida y temperatura como funciones numéricas nos permiten concluir que puede existir una estrecha relación entre ambos indicadores.
\end{abstract}

Palabras clave: expansión urbana, descomposición modal empírica, incremento de temperatura, optimización por enjambre de partículas.

\section{Analysis of the Relation between Urban Growth and Temperature Increment in Merida City using Satellite Images}

Abstract. In this article an analysis of the correlation between the growth of urban sprawl and the change of temperatures of the city of Merida, Yucatan, 


\begin{abstract}
Mexico, is made by means of the implementation of artificial intelligence techniques focused on the segmentation of images. Starting from a multitemporal sequence of satellite images registered by Landsat in RGB format in a period from 2001 to 2016, the segmentation of the urban spot is first performed using an artificial intelligence technique, particularly particle swarm optimization, an implementation of swarm intelligence. The segmentation of the data allows us to estimate the built-up area in the city. Later, the historical data of temperatures registered in that same period are analyzed with the method of empirical mode decomposition. The preliminary analysis of the positive correlation between the data of built-up area and temperature as numerical functions allows us to conclude that there may exist a close relationship between both indicators.
\end{abstract}

Keywords: urban expansion, empirical mode decomposition, temperature increase, particle swarm optimization.

\title{
1. Introducción
}

Diversos estudios han comprobado que la sustitución drástica de los ecosistemas naturales por elementos urbanos (pavimento, asfalto, etc.) altera el clima local y de la región ya que el balance de energía se altera [1]. En este sentido, el clima urbano es el resultado del efecto de la radiación solar que reciben las superficies de la ciudad y que posteriormente es remitida a la atmósfera. Esto último sucede a través de mecanismos de calentamiento del aire, de evapotranspiración de la vegetación y todo aquel calor almacenado en las superficies urbanas. La evapotranspiración en las ciudades se reduce de manera abrupta debido a que las áreas húmedas son muy escasas, además de que los materiales de construcción no cambian sus propiedades térmicas, es decir, la cantidad de energía que almacenan es constante. Como consecuencia, el caldeamiento del aire cercano a la superficie del suelo aumenta generando el fenómeno de la isla de calor urbana (ICU) que se caracteriza principalmente porque la temperatura del aire es más alta en el área urbana que en los alrededores rurales, y que se puede considerar como un cambio climático local o regional [1].

Las variaciones en las temperaturas extremas son de particular importancia debido a su relación con la biodiversidad, así como con diversas actividades humanas como la agricultura, ganadería y la demanda de energía. Un ejemplo, en los años de 1906 a 2005 , el aumento en la temperatura terrestre en promedio se estimaba en $0.74 \pm 0.18$ ${ }^{\circ} \mathrm{C}$; aunque el valor no es grande, se observaron efectos visibles en muchos sistemas físicos y biológicos [2].

La ciudad de Mérida, Yucatán, México, localizada en las coordenadas 20,9667 de latitud Norte y $-89.6167^{\circ}$ de longitud Oeste, es el caso de estudio de la presente investigación debido al ritmo de crecimiento acelerado que ha presentado durante los últimos años. Por mencionar algunos datos estadísticos, en el año de 1950 la mancha urbana era de 4,264 ha, con una población aproximada de 208,620 habitantes; para 1978 la mancha creció hasta alcanzar 7,313 ha y una población de 424,500; en el año 1998 la ciudad ocupó un área de 15,944 ha con 705,100 habitantes; en 2010 la mancha ocupó 27,027 ha con una población de 870,084 habitantes [3]. La notable expansión territorial de las últimas décadas muestra que el área urbana ha crecido en promedio 
Análisis del crecimiento urbano y su relación con el incremento de temperaturas en la ciudad...

alrededor de $80 \%$ respecto de la década de los 80 s; la superficie conurbada de Mérida aumentó a un ritmo anual promedio de $4.42 \%$ en 30 años (1990-2010), mientras que la población en la misma área creció a un ritmo menor de $2.26 \%$ anual [4]. Por lo anterior, todo el ritmo de crecimiento acelerado conlleva a que se comiencen a sentir los efectos de las islas de calor urbano.

Algunos estudios que marcan la relación entre el cambio climático regional por consecuencia de la mancha urbana son los siguientes. En [5], se analizan las tendencias anuales de temperaturas extremas para la ciudad de Mexicali, Baja California, México, mediante una serie de tiempo de 1950 a 2010; los autores concluyen que hacia finales del siglo XXI la temperatura máxima extrema podría ser de 2 a $3{ }^{\circ} \mathrm{C}$ más alta que la actual, ya que el modelo probabilístico empleado sugiere incrementos de 7 a $9{ }^{\circ} \mathrm{C}$ en la temperatura mínima extrema respecto al periodo de base estudiado. En [6] se analizó la intensidad del efecto de isla de calor urbana y el efecto de la cobertura vegetal sobre la regularización de la temperatura del aire.

Para el estudio se definieron cuatro zonas climáticas locales en la ciudad de Querétaro, Querétaro, México: tres urbanas y una rural. La temperatura del aire se midió con recolectores de datos a intervalos de 30 minutos entre junio de 2012 y mayo de 2013; además se analizaron datos climáticos de seis estaciones meteorológicas. Los autores concluyen que una mayor cobertura de la vegetación mejora las condiciones ambientales en términos de humedad relativa y regularización de los extremos de temperatura durante la temporada cálida.

En este trabajo de investigación se realiza un análisis de la correlación entre el área construida de la ciudad de Mérida y el cambio de temperaturas. Para ello, se realiza el análisis de una serie de imágenes satelitales registradas por las diferentes misiones Landsat, comprendido entre el 2001 al 2016. La organización de este trabajo es como sigue. La Sección 2 presenta las técnicas y los algoritmos utilizados en esta investigación. En la Sección 3 se discute la metodología propuesta y se presentan los resultados. Finalmente, la Sección 4 describe las conclusiones de este trabajo.

\section{Materiales y métodos}

Para la segmentación de la mancha urbana o área construida de la ciudad de Mérida se utilizó una serie multitemporal de imágenes registradas por los satélites Landsat 5 (Thematic Mapper, TM) y 8 (Operational Land Imager and Thermal Infrared Sensor, OLI-TIRS), las cuales son de libre acceso. Cada una de las bandas espectrales de ambas misiones tiene una resolución espacial de $30 \mathrm{~m}$ por pixel.

Las imágenes analizadas fueron registradas en el periodo que comprende del 2001 al 2016 y se seleccionaron aquellas con la menor cobertura de nubes posible (menor al $10 \%$ ). De esta manera, a cada imagen se le realizó una corrección atmosférica y se obtuvo una imagen en color RGB producida por la combinación de las bandas 3, 2, 1 para TM y 4, 3, 2 para OLI-TIRS, respectivamente (ver Fig. 1). 

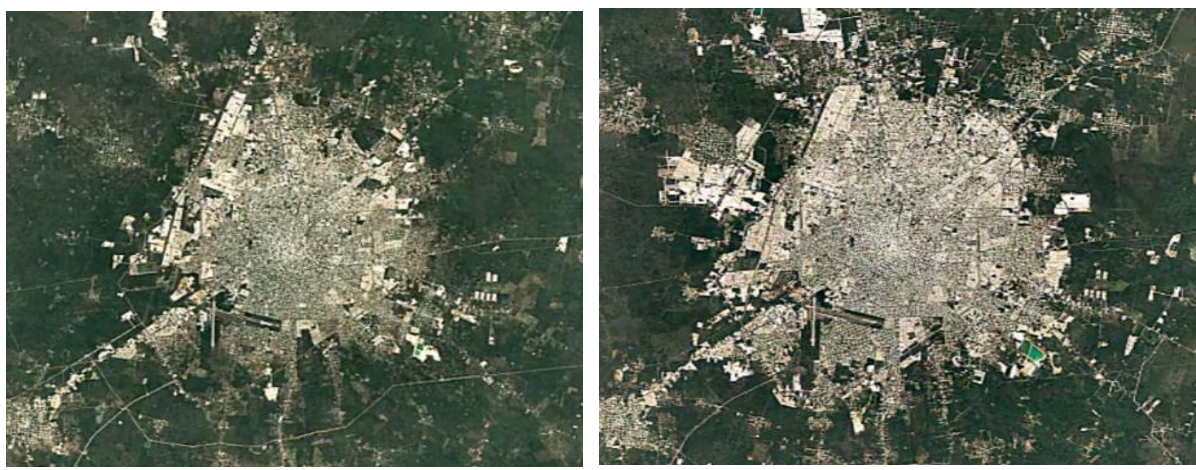

Fig. 1. Imágenes en color RGB de la ciudad de Mérida registradas en 2001 (izquierda) y 2016 (derecha).

\subsection{Optimización por enjambre de partículas}

Para la segmentación de las imágenes se utiliza una variante de la técnica de inteligencia de enjambres, una subárea de la inteligencia artificial, llamada optimización por enjambre de partículas (OEP) (particle swarm optimization), la cual se describe a continuación. Una herramienta matemática muy útil en ciencias aplicadas es la teoría del cálculo fraccional (CF). La CF ha jugado un rol muy importante en incrementar el desempeño de algoritmos utilizados en modelado, funciones de curva, filtrado, reconocimiento de patrones, detección de bordes, identificación, estabilidad, control, observación, robustez, etc. Se propone el uso de OEP con enfoque Darwiniano en combinación del uso de un orden fraccional (OF), dando como resultado un algoritmo conocido comúnmente como optimización Darwiniana por enjambres de partículas con orden fraccional (ODEPOF).

El principio de esta función es el siguiente: cada partícula o individuo tiene una posición (que en dos dimensiones está determinada por un vector) en el espacio de búsqueda y una velocidad (como otro vector), con la que se mueve a través del espacio. Además de la posición y velocidad, estas partículas presentan una inercia que los mantiene en la misma dirección del movimiento original, y una aceleración (o cambio de velocidad con respecto al tiempo), misma que depende de dos características principalmente: cada partícula es atraída hacia la mejor localización que ha encontrado 1) esta misma en su historia (mejor individual), y 2) el conjunto de partículas en su totalidad en el espacio de búsqueda (mejor global).

Las fuerzas que empujan a las partículas en cada una de estas dos direcciones pueden ajustarse de tal forma que a medida que las partículas se alejan de estas localizaciones, la atracción es mayor [7]. Un factor aleatorio que influye en cómo las partículas son impulsadas hacia estas localizaciones es también incluido.

Esta herramienta es de suma importancia para el análisis de nuestras imágenes, ya que al aplicar este algoritmo se puede realizar la división de las áreas verdes y área urbana, realizando la segmentación de los elementos que se quieren cuantificar. 


\subsection{Descomposición modal empírica}

Para continuar con nuestro estudio, además de las imágenes satelitales, se obtuvo el registro del historial de temperaturas del periodo 2001-2016. Estos datos son muy relevantes para esta investigación, ya que nos ayudarán a crear datos estadísticos que serán los que darán a conocer los resultados y comprobar si existe un notable cambio climático en la región.

Los datos obtenidos de las imágenes procesadas y del historial de temperaturas de la región se utilizaron para generar gráficas cuya tendencia fue extraída mediante el método de descomposición modal empírica (DME), sobre las cuales es posible entonces hacer el cálculo del coeficiente de correlación entre el crecimiento de la mancha urbana y las temperaturas de la región.

El algoritmo de DME, presentado por primera vez en 1998 [8], se basa en producir envolventes lisos definidos por máximos y mínimos locales de una secuencia y la subsecuente substracción de la media de estas envolventes a partir de la secuencia inicial. Esto requiere de la identificación de los extremos locales que están conectados por líneas spline cúbicas para producir los envolventes superior e inferior [9].

Es un método adaptivo de análisis adecuado para el procesamiento de series que son no estacionarias y no lineales. La DME realiza operaciones que dividen una serie en funciones modales intrínsecas (FMIs) sin salir del dominio del tiempo. Se puede comparar con otros métodos de análisis frecuencial como la transformada de Fourier y la descomposición de ondas. La DME ha sido ampliamente aplicada en distintos campos de la ciencia con fines de reconocimiento [10], análisis [11], filtrado[12], predicción [13], etcétera. El método fue propuesto como la parte fundamental de la transformada Hilbert-Huang (THH).

La aplicación de la DME, de manera general, consiste en localizar los valores máximos y mínimos de la señal, conectar máximos y mínimos, respectivamente, mediante un spline cúbico para obtener envolventes superior e inferior. La media de ambas envolventes es un prototipo de la primera FMI. Este último paso se repite iterativamente hasta que la salida sea una señal con media cero, de acuerdo con un criterio de convergencia. En contraste con la transformada de Fourier y las ondeletas (wavelets), la DME descompone cualquier dato dado en FMIs que no se establecen analíticamente y en su lugar se determinan sólo mediante una secuencia analizada.

\section{Metodología y resultados}

Se analizaron 16 imágenes satelitales de la ciudad de Mérida correspondientes a los años 2001-2016; estas imágenes se muestran en la Fig. 2. En estas imágenes es evidente cómo la mancha urbana ha desplazado las áreas verdes mediante distintos tipos de construcciones, tanto residenciales como industriales. 


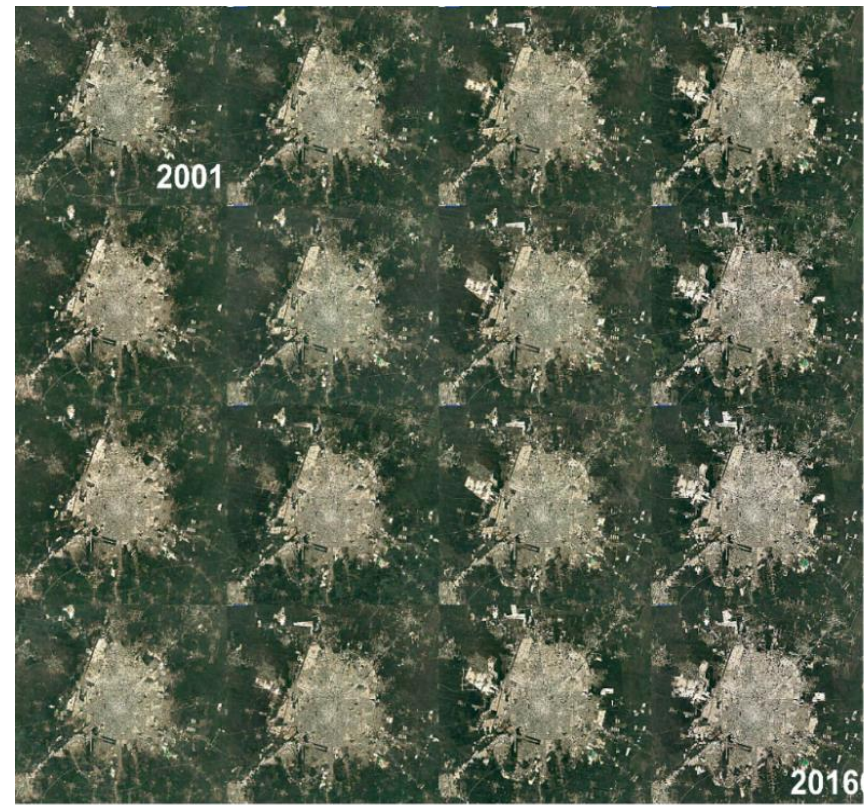

Fig. 2. Imágenes satelitales en RGB de la ciudad de Mérida, Yucatán, México, entre los años 2001 (imagen superior izquierda) y 2016 (imagen inferior derecha).

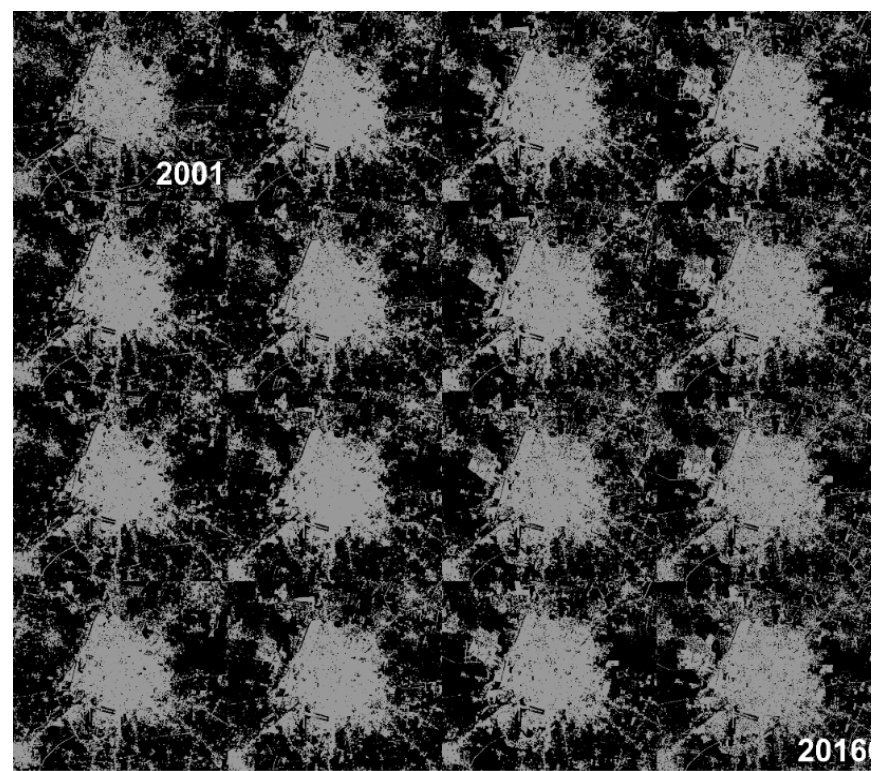

Fig. 3. Imágenes resultantes de la segmentación de las imágenes satelitales de la ciudad de Mérida, Yucatán, México, entre los años 2001 (imagen superior izquierda) y 2016 (imagen inferior derecha) mostradas en la Fig. 2. La mancha urbana se despliega en color gris (por motivos visuales), mientras que otras coberturas terrestres (vegetación, cuerpos de agua, sembradías, entre otros) se muestras en color negro. 
A partir de la versión RGB de las imágenes se aplicó un algoritmo de segmentación de imágenes basado en la OEP (Sección 2.1), lo que permitió segmentar el conjunto de imágenes mostrado en la Fig. 2, y obtener el conjunto de imágenes segmentadas mostrado en la Fig. 3.

El conteo de los pixeles segmentados de las imágenes de la Fig. 3 permite realizar una aproximación del área correspondiente a la mancha urbana durante cada uno de los años comprendidos entre 2001 y 2016. Con esta información se realizó la gráfica mostrada en la Fig. 4. Los datos correspondientes a las áreas de mancha urbana son consistentes con los datos reportados en documentos del gobierno estatal [3]. Para determinar la tendencia de estos datos se utilizó el método descrito de DME. De esta manera, la descomposición asociada a la frecuencia más baja (la tendencia general de los datos) se muestra sobrepuesta a los datos obtenidos por el algoritmo de segmentación OEP.

Una vez obtenida la función numérica de tendencia utilizando la técnica de DME, es necesario correlacionarla con la función numérica equivalente a las temperaturas históricas reportadas en la ciudad de Mérida entre los años 2001 y 2016 [14]. Debido a su evidente comportamiento creciente en este intervalo de tiempo, se utilizaron los datos correspondientes a la temperatura mínima mensual, que se muestran en la Fig. 5 mediante una línea azul semi-continua. La tendencia de estos datos correspondiente a la descomposición de frecuencia más baja utilizando la técnica de DME se muestra sobrepuesta con una línea roja continua.

Ambas funciones numéricas que representan la tendencia tanto de los datos del área de la mancha urbana, como de las temperaturas mínimas, durante los años 2001-2016, muestran un comportamiento creciente. Cualquier hipótesis que relacione el crecimiento de las temperaturas como una consecuencia del crecimiento de la mancha urbana implica que estas funciones deben mostrar cierta correlación estadística. El cálculo del coeficiente de correlación de Pearson, ecuación (1), para las funciones de tendencia de temperaturas mínimas mensuales y de crecimiento de la mancha urbana, se calculó acorde a la ecuación:

$$
\rho_{f, g}=\frac{\operatorname{cov}(f, g)}{\sigma_{f} \sigma_{g}},
$$

donde $\rho_{f, g}$ representa el coeficiente de correlación de las funciones $f$ (área de mancha urbana) y $g$ (temperaturas mínimas mensuales), $\operatorname{cov}(f, g)$ la covarianza de las funciones $f$ y $g$, y $\sigma$ la desviación estándar de una función dada. El valor para el coeficiente de correlación de Pearson dio como resultado 0.495, mostrando la correlación positiva y consecuentemente una estrecha relación entre los fenómenos de crecimiento de la mancha urbana y del incremento de las temperaturas en la ciudad de Mérida.

\section{Conclusiones}

La ciudad de Mérida, Yucatán, México, ha sido sujeta a un considerable crecimiento de la mancha urbana en los últimos años, lo que implica también una pérdida de áreas 


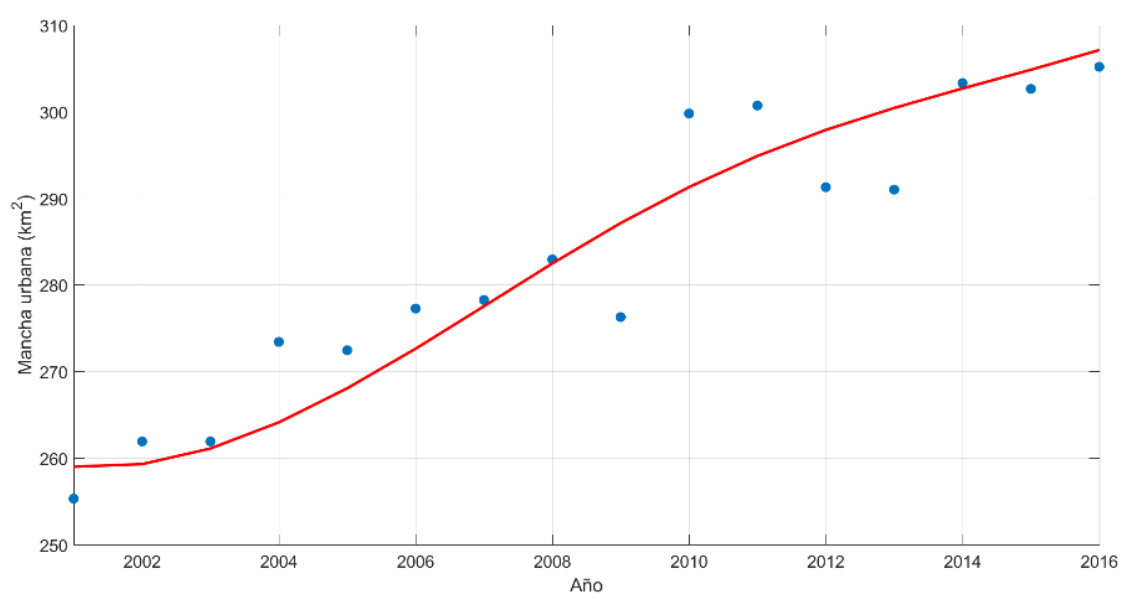

Fig. 4. Los datos correspondientes al área de la mancha urbana detectada en la ciudad de Mérida, Yucatán, México, mediante el algoritmo de segmentación de imágenes basado en OEP, mostrados en kilómetros cuadrados. Los datos obtenidos por el algoritmo se muestran utilizando los puntos azules, mientras que la línea roja continua describe la tendencia de estos datos mediante la descomposición de frecuencia más baja utilizando la técnica de DME.

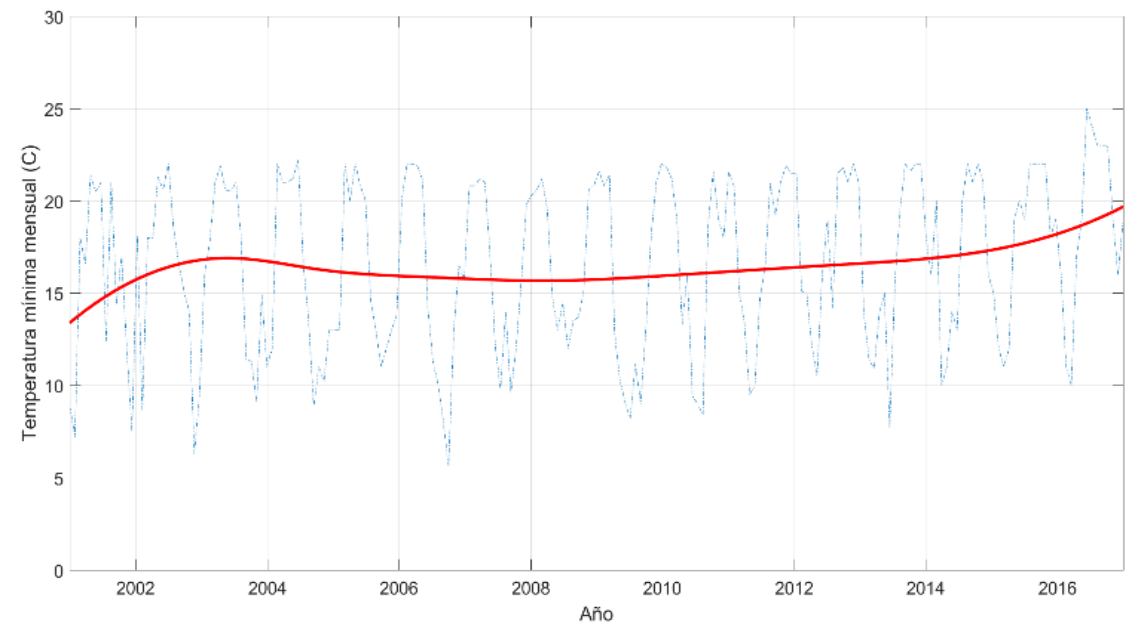

Fig. 5. Los datos correspondientes a la temperatura mínima mensual presentada en la ciudad de Mérida, Yucatán, entre los años 2001-2016 (línea azul semi-continua). Se muestra también sobrepuesta la tendencia de estos datos correspondiente a la descomposición de frecuencia más baja utilizando la técnica de DME (línea roja continua).

verdes. Este fenómeno puede estar relacionado con el incremento de las temperaturas mínimas mensuales en años recientes. 
En este trabajo se propone un algoritmo de segmentación de imágenes basado en IA, particularmente OEP, para extraer las áreas correspondientes a la mancha urbana de una serie de imágenes satelitales de la ciudad de Mérida, entre los años 2001 y 2016, y así poder cuantificar su crecimiento. Los datos obtenidos por el algoritmo de segmentación son consistentes con reportes gubernamentales.

Después de obtener las tendencias en el crecimiento tanto de la mancha urbana como de las temperaturas mínimas mensuales mediante el método de DME, se calculó el coeficiente de correlación entre ambas funciones obteniendo un valor de 0.495. La correlación positiva confirma que estos dos fenómenos se encuentran relacionados entre sí, y puede ser un primer paso para la determinación de la posible causalidad entre ambos fenómenos, lo que permitiría establecer líneas prioritarias de acción para el control de la mancha urbana en la ciudad.

Agradecimientos. Se agradece al Tecnológico Nacional de México/I.T. Mérida, por el apoyo económico mediante los proyectos 6513.18-P y 6511.18-P.

\section{Referencias}

1. Barradas, V. L.: La isla de calor urbana y la vegetación arbórea. Oikos 7, pp. 14-16 (2013)

2. IPCC: Cambio climático 2007: Informe de síntesis. Grupo Intergubernamental de Expertos sobre el Cambio Climático (2007)

3. SEDUMA: Crecimiento de la mancha urbana (1950-1978-1998-2010), http://www.seduma.yucatan.gob.mx/desarrollo-urbano/documentos/ZonaMetropolitana/1_ 3_Crecimiento_Urbano.pdf (2018)

4. PIDEM: Programa Integral de Desarrollo Metropolitano de Mérida (PIDEM), http://www.fpeyucatan.org.mx/wp-content/uploads/PDF/PIDEM (2018)

5. García-Cueto, O.R., Santillán-Soto, N., Quintero-Nuñez, M., Ojeda-Benítez, S., VelázquezLimón, N.: Extreme temperature scenarios in Mexicali, Mexico under climate change conditions. Atmósfera 26(4), pp. 509-520 (2013)

6. Colunga, M.L., Cambrón-Sandoval, V.H., Suzán-Azpiri, H., Guevara-Escobar, A., LunaSoria, H.: The role of urban vegetation in temperature and heat island effects in Querétaro city, Mexico. Atmósfera 28(3), pp. 205-218 (2015)

7. Sancho-Caparrini, F.: PSO: Optimización por enjambres de partículas. (2018)

8. Huang, N. E., Shen, Z., Long, S. R., Wu, M. C., Shih, H. H., Zheng, Q., Yen, N. C., Tung, C. C., Liu, H. H.: The empirical mode decomposition and the Hilbert spectrum for nonlinear and non-stationary time series analysis. Proceedings Mathematical, Physical and Engineering Sciences 454(1971), pp. 903-995 (1998)

9. Kim, D., Oh, H.: EMD: A Package for Empirical Mode Decomposition and Hilbert Spectrum. The R Journal, pp. 40-46 (2009)

10. Du, H., Cao, J., Xue, Y., Wang, X.: Seismic facies analysis based on self-organizing map and empirical mode decomposition. Journal of Applied Geophysics 112, pp. 52-61 (2015)

11. Nunes, J., Bouaoune, Y., Delechelle, E., Niang, O., Bunel, P.: Image analysis by bidimensional empirical mode decomposition. Image and Vision Computing 21(12), pp. 1019-1026 (2003)

12. Andrade, A. O., Nasuto, S., Kyberd, P., Sweeney-Reed, C.M., Van Kanijn, F. R.: EMG signal filtering based on Empirical Mode Decomposition. Biomedical Signal Processing and Control 1(1), pp. 44-55 (2006) 
Saul Navarro-Tec, Mauricio Gabriel Orozco-del-Castillo, Juan Carlos Valdiviezo-Navarro, et al.

13. Drakakis, K.: Empirical mode decomposition of financial data. International Mathematical Forum 3(25) pp. 1191-1202 (2008)

14. Histórico del clima en Mérida, https://www.meteored.mx/clima_Merida-America+NorteMexico-Yucatan-MMMD-sactual-22381.html\# (2018) 